The method of aralysis which Prof. Lewes finds best is as follows :-

Two of Stead's apparatus are taken and placed with the entrance tubes end to end, and filled-one with distilled water saturated with air, and the other with clean pure mercury. The gas to be tested is collected in one of the Stead absorbing tubes, over water, so as to be saturated; it is then transferred over mercury in the eudiometer tube of the second apparatus, and measured and passed into sodic hydrate, in order to absorb the small trace of carbon dioxide to be found in the highly-purified London gas. When present in only small traces, the amount of carbon dioxide lost by water saturation cannot be detected. After the absorption of the carbon dioxide, the gas is run into the second apparatus, and the oxygen estimated by absorption with alkaline pyrogallate, which must be strong and fresh, containing about 25 grammes of pyrogallic acid dissolved in 50 grammes of sodic hydrate in 200 c.c. of water. It is absolutely essential that the solution should be fresh, as after some time it will evolve a considerable amount of carbon monoxide. The heavy hydrocarbons have now to be estimated; and inasmuch as benzene is one of the most valuable illuminants in the coal gas, it would be of great value if any absorbent could be found that would separate the benzene and ethylene series. Unfortunately this does not exist as far as is known; the usual absorbents having the following drawbacks :- (I) Nordhausen sulphuric acid, in which sulphur trioxide has been dissolved until it will solidify on cooling, absorbs both ethylene and benzene, and therefore cannot be used to separate them. (2) Fuming nitric acid is a good absorbent for both series. (3) Bromine water acts far more rapidly on ethylene than on benzene, but undoubtedly does absorb a considerable quantity of the latter if left long in contact with a mixture of the two. (4) None of the foregoing affect methane in diffused daylight. The nearest approximate result is obtained by treating the gas first with strong bromine water, but not leaving it too long in contact with it, and then removing bromine vapour over sodic hydrate-the absorption being taken as the ethylene series while the benzene is absorbed by fuming nitric acid or saturated Nordhausen acid-acid fumes being removed in the sodic hydrate tube before measurement over water. After absorption with nitric acid gas, is run back into the eudiometer, and measured over water. It is then passed into an absorption tube filled with a fresh solution of ammoniacal cuprous chloride. This must not be used for more than six determinations of an ordinary coal gas containing (say) 3 to 6 per cent. of carbon monoxide, or 3 of a carburetted water gas, as, after much carbon monoxide has been absorbed, the solution has a tendency to again give up small quantities of the gas. The gas is now returned to the mercury eudiometer tube ; and, after measurement, it is passed into an absorption tube containing ordinary paraffin oil (previously heated until everything that will distil at $100^{\circ} \mathrm{C}$ has gone off), which absorbs ethane, propane, butane, and a good deal of the methane. The residue is then washed and mixed with oxygen, which has itself been analysed, so that the percentage of nitrogen and foreign gases in it is known, and the mixture exploded over mercury. The carbon dioxide formed is estimated; and its volume plus the volume of gas absorbed by the paraffin gives the volume of gases in the methane series. A fresh portion of gas is now taken over mercury, and is exploded with excess of analyzed oxygen. The carbon dioxide is absorbed by sodic hydrate, and the oxygen by pyrogallate; and the residue will be the nitrogen - the hydrogen being determined by difference. In this way an analysis of South Metropolitan gas shows-

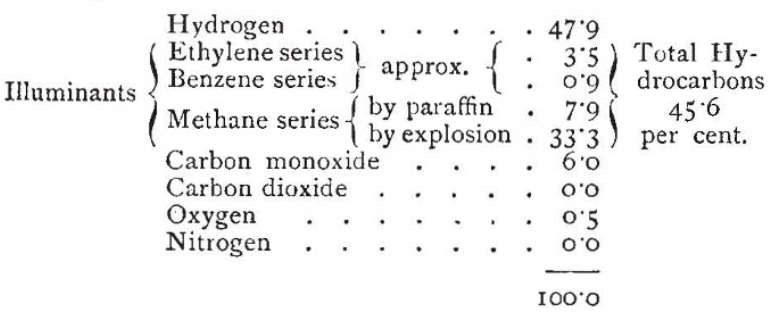

In such an analysis, the lecturer remarked, no pretence was made that the exact percentage of each illuminant was given, but the total of the bydrocarbons is accurate; and their

NO. I IO6, VOL. 43] rough subdivision gave a far clearer insight into the characters of the gas than the more pretentious and more faulty analysis upon which it has been customary to argue. He said it must be clearly borne in mind that he only put forward this scheme of analysis to meet the need now rapidly arising for a method which would show whether ordinary coal gas enriched by cannel, coal gas carburetted with either gasoline or oil gas, or coal gas enriched by highly carburetted water gas, was being dealt with. In the first case, the ethylene and benzene series would be found well represented, while the carbon monoxide was low; in the second, the amount of hydrocarbons in the methane series would have become greater, and if oil gas had been used, a small increase in carbon monoxide might also be noticed; while the presence of carburetted water gas at once brought up the quantity of carbon monoxide, and the methane series became more important illuminants.

Prof. Lewes went on to show that the light-giving value of the hydrocarbons present in coal gas varies very greatly, the illuminating power increasing very rapidly with the number of carbon atoms in the molecule ; and concluded by fully discussing the effect which the various diluents present in coal gas had upon its illuminating value.

(To be continued.)

\section{ON THE ORBIT OF a VIRGINIS.}

PROF. II. C. VOGEL has contributed a further discussion o the orbit of a Virginis (Spica) to the Astronomische Nachrichten, No. 2995. The following is a translation of the greater part of his paper :-

"After the periodical approach and retreat of this star, which was suspected from last year's observations, had been proved by some spectrographic observations made this year, an examination of the spectrum has been made at every favourable opportunity. So far, it has been possible to ohserve Spica on twenty-four evenings, thus affording material which allows its period to be determined with a greater degree of accuracy. Before communicating the results of the measurements of the photographs it will be necessary to preface a few remarks relative to the accuracy attainable.

"In No. 2896 of the Astronomische Nachrichten I detailed the first results which were obtained by means of the new spectrograph. The measurements were then made on some stars with spectra of the second class, and the increased accuracy given to these observations by the fact that the measurements were not made on the $\mathrm{H} \gamma$ line only, but on some exceedingly sharp lines in the neighbourhuod, has been fully confirmed by the now completed measurements of all the stars of the second and third class accessible to the Potsdam instruments.

"In the case of stars of class I. $a$, however, in which the $\mathrm{H} \gamma$ line is more or less broad and fuzzy at the edges, and there are no other lines near it, greater difficulties than I at first expected opposed themselves to the satisfactorily exact measurement of their spectra. At one time I had the intention of making the measurements, not on the hydrogen line, but on the better defined lines of another metal, such as magnesium, which possesses a strong line at $448 \mu \mu$. I abandoned this intention, however, because by adopting this means a number of stars, in whose spectra the said line of magnesium is weak or invisible, must have been excluded.

"The absorption lines of hydrogen in star spectra show by their appearance the following four types :-

" (a) Dark lines, with somewhat undefined edges, e.g. Capella.

“(b) Broad dark lines, with somewhat undefined edges, e.g. Rigel.

" $(c)$ Broad bands, gradually fading away, but with a more or less broad and well-defined maximum of intensity in the centre, e.g. Sirius.

" (d) Broad bands, undefined at the edges, without any remarkable maximum of intensity in the centre (Spica).

"The four types are graphically represented by the curves in the accompanying figure."

Prof. Vogel notes that when the maximum of intensity of the form $(c)$ lies outside the line of comparison, the measurement of the distance between the two is attended with no special difficulties. When, however, the comparison line overlaps the line in the star spectrum under examination, or in the case of spectra of the form $(d)$, special devices have to be used to determine the distance 
from the centre of the absorption line to the line of comparison, due to the star's motion in the line of sight. These methods
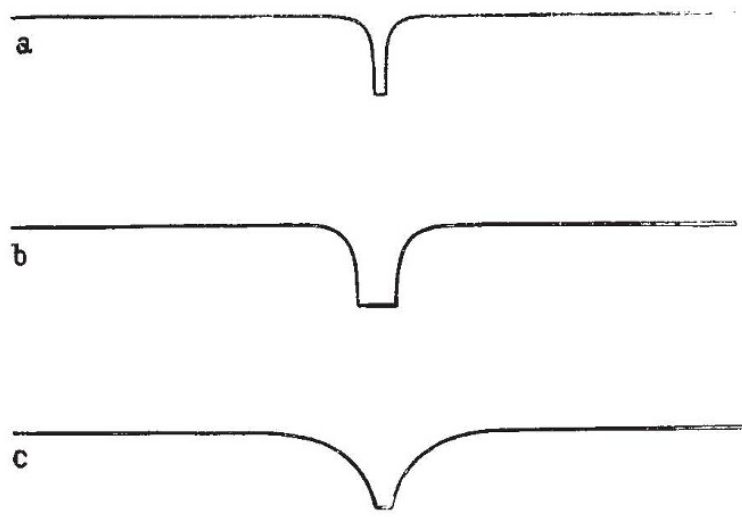

d

Diagrammatic representation of the appearance of hydrogen absorptionlines in four types of stellar spectra.

are described in the paper. The values obtained from measurements of photographs of the spectrum of Spica are shown in the following table :-

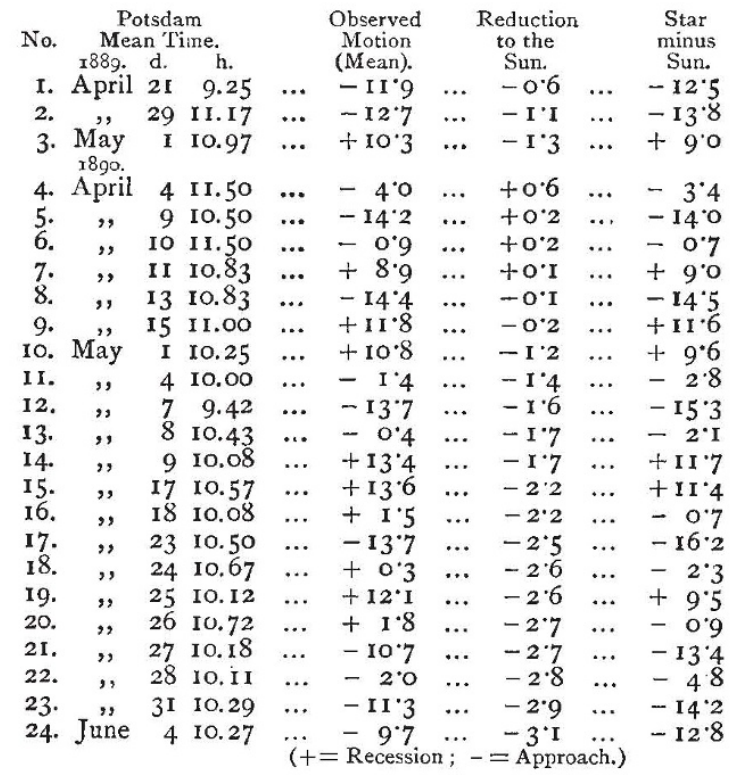

With regard to these results, Prof. Vogel remarks:-

"If an early circular orbit be accepted, the observations here aescribed allow of its possessing the form and period given by me in my first communication on a Virginis (Sitzungsber. $d$. Akad. d. Wissensch. zu Beriin, April 24, 1890), and it is shown that the period of revolution of 4.oII days is a safe one for a year's time, for the observations of this year are not in accordance with the epochs computed from the periods 3.967 and 4.055 days. Only a slight increase of the first period, 4 or I days, is indicated by the better reduction of last year's observations. The following elements have been deduced from the collected material :-

"Epoch $t_{0}$ (when the total orbital motion of the two components in the line of sight $=0)=1890$ May $4 \mathrm{~d}$. 10. $5 \mathrm{oh}$. Potsdam mean time.
"Period $p=4$ "o134 days.

"Motion of the star system $=-2 \cdot 0$ geographical miles.

"Velocity in the line of sight, when the translation of the system has been deducted, $=12 \cdot 3 \sin \left(\frac{t-t_{0}}{p} 360^{\circ}\right)$ geographical miles.

"The difference of four miles between the mean maximum positive and negative velocities might be explained by the hypothesis that the orbit of the star deviates considerably from a circle, and that the major axis of the ellipse is almost perpendicular to the line of sight. The observations, however, are not sufficient to decide this point, and as the motion of the system of $-2{ }^{\circ}$ o miles (obtained by taking a circular orbit) corresponds to the average movement arrived at from the Potsdam observations, no foundation is afforded for any other hypothesis. ... In consequence of my first communication on the orbit of $\alpha$ Virginis, Prof. Bakhuyzen has examined whether the Greenwich observations, notwithstanding their very slight accuracy, would.afford data for a somewhat more exact determination of the period, as they extend over several years; and he has arrived at a period of 4 days 0.357 hours. From further communications I have learned from Prof. Bakhuyzen that his determination only rested on the comparison of some observations made at Greenwich in the years 1883 and 1886 with the Potsdam observations for the same years, but that no comparison of all the observations had been made. A somewhat more exact repetition of the calculation with respect to the times of observation, has, however, shown that, in consequence of the unaccustomed reckoning of time from midnight to midnight, which has been in use at Greenwich since 1885 , Prof. Bakhuyzen had counted an observation as having been made on May 4, 1886, whereas according to astronomical reckoning it was made on May 3. When this oversight is corrected, the period obtained was 4 days 0.324 hours. I mention this, because Mr. Christie, in his annual report for 1890 , has referred to the above-named period of 4 days 0.357 hours.

"I have examined the Greenwich observations somewhat care fully, and have come to the conclusion that they cannot be looked upon as sufficient to contradict the results of the Potsdam observations, and that no correction to the discovered period can be deduced from them with any certainty."

After a statement of the results of twenty-six observations of $\alpha$ Virginis made at Greenwich between 1876 and 1889 , for motion in the line of sight, Prof. Vogel remarks :-

"If, taking twenty-six observations of $\alpha$ Virginis, the average result be counted, and no orbit introduced, it amounts to \pm 5.8 geographical miles. In the case of $a$ Leonis it is $\pm_{4} .4$, of $\alpha$ Ophiuchi \pm 6.7 , and of a Aquilæ \pm 6.5 (putting on one side the observations of 1874,1875 , and 1876 , which give the value of \pm 4.5 miles). Hence it is seen that, according to the Greenwich observations, there is no more cause for suspecting a periodical change of motion for $\alpha$ Virginis than for the other stars examined.

"If values are computed back to $\mathbf{I} 886$ from the elements of the orbit previously given, a minimum of motion in the line of sight is obtained for 1886 April $30,13.12$ hours, and a maximum of negative motion on May $3,13.36$ hours, Potsdam time. These results agree very well with the Greenwich observation of those days. From the interval of time between the two minima in 1886 and in 1890 , periods of 3.970 days and $4^{\circ} 05^{\circ}$ days were obtained. With these two periods, and that best represented by the Potsdam observations, viz. 4.0134 days, I have computed some of the Greenwich observations according to the formula $12.3 \sin \frac{t-t_{0}}{p} 360^{\circ}$, after the translation of the system deduced from the Potsdam observations had been subtracted. It did not seem allowable to carry the reckoning any further back, as even the period of $4^{\circ}$ O134 days may be doubtful in the third decimal place."

A comparison of nine observations made between April 1886 and May 1889 with calculated motions based on the assumed period of 3.970 days, gave a residual of \pm 4.4 ; with the period $4^{\circ} \mathrm{Or} 34$ days it amounts to \pm 6.6 ; and with the period $4.05^{8}$ days reaches \pm 8.5 . If the star be considered to have no orbital motion, the residual error is \pm 7.2 miles. It appears, therefore, that the Greenwich observations of I886 to 1889 are best represented by the period 3.970 days. Prof. Vogel then goes on to say :-

"I repeat, with the new values, the calculation of the extent

No. IIO6, VOL. 43] 
of the orbit and the mass of the stars, which I gave in my first communication on this subject. Upon the supposition of a circular orbit, the resulting period is $4^{\circ} 0134$ days, while the two components have equal masses and a velocity of 12.3 miles per second. The mass of the system is 2.6 that of the sun, and the distance of each component from the common centre of gravity is 679,000 geographical miles. With a parallax of $\mathrm{o}^{\prime \prime} \cdot 2$ the maximum apparent distance amounts to $\mathrm{o}^{\prime \prime} \cdot \mathrm{O} 14$, so that the satellite cannot be seen with the strongest instrument.

"Finally, I must not omit to say that, by means of repeated examinations of the photographic plates and measurements of the spectrum, it appears more and more certain that the satellite of $\alpha$ Virginis has made itself apparent on the impressions. On several plates, taken at the time of maximum movement, one edge of the $\mathrm{H} \gamma$ line seems to be rather more undefined and more gradually to diminish in intensity than the other edge; also on some other plates, taken at the time of minimum motion, the $\mathrm{H} \gamma$ line seems to be rather narrower. These plates also show other lines in the spectrum more plainly on account of their being less faint. These appearances go to show that the satellite has a similar spectrum to the primary star, and that the $\mathrm{H}_{\gamma}$ line is also broad and faint, but so faint in comparison to the line in the spectrum of the primary star that its presence is only suspected after most careful examinations of the photographs. The phenomenon observed has no influence on the measurements. If, however, the spectrum of the satellite were stronger, it might exercise an influence on the measurements of the maximum motion by causing the differences to measure somewhat less than they really are.

"The satellite, if we grant that it really exists, is probably of about the third magnitude, and it may be possible that powerful reflectors may demonstrate its presence by showing more distinctly the slight periodic changes in the composite spectrum."

\section{SOCIETIES AND ACADEMIES.}

LONDON.

Royal Society, December I I, I89o.-- " Photometric Observations of the Sun and Sky." By William Brennand. Communicated by C. B. Clarke, F.R.S.

The paper begins with a short account of the various papers communicated by Sir H. Roscoe, and published in the Transactions of the Royal Society.

My observations were made at Dacca, East Bengal, in 186r66, repeated at Milverton, in Somersetshire, during the last two years. My first experiments were directed to ascertaining the action of the sun on sensitized paper exposed at right angles to the solar rays for different altitudes of the sun, and largely to ascertaining the laws of distribution of the actinic power in the sky.

I take no observations except when the sky is quite clear.

The method of measurement I adopted is the darkening produced in sensitized paper. I cut strips from one uniform sheet of ordinary photographic paper. My observations being relative, I obtain the same results (ratios) with any paper. I compare ultimately the effects of the sun and of a candle on this same paper.

I assume that, in burning a stearine candle, the chemical action is proportional to the material consumed; I have taken as my unit $(i)$ of measure of chemical action the darkening produced at a distance of $\mathbf{I}$ inch from the wick of the candle when roo grains were consumed, which in the candle I used in India occupied about forty-seven minutes. My observations, being almost entirely relative, are independent of these assumptions, which affect hardly any of my results except comparisons with the absolute unit measures of Sir H. Roscoe.

The water-motion actinometer, with which observations of the action of sun and sky were made, is the instrument depicted in the photograph (see figure).

[The water-motion actinometer was shown and explained. By it, a shutter is removed with uniform velocity from a strip of sensitized paper, which is thus, by any light to which it is exposed, tinged with a gradually intenser depth from o to 16 seconds (the paper being suddenly covered at the end of 16 seconds). If, for example, at a particular altitude of the sun, the action of the sky alone in ' 6 seconds produced the same depth of tint that the action of the sun alone produced in ro seconds

NO. I IO6, VOL. 43] (in a similar strip cut from the same paper), then I estimate the chemical effect of the sun to have been $\frac{6}{10}$ that of the sky, i.e. it would be 0.6 if the effect of the sky was taken as the unit of measurement. In the same unit, the effect of the sun and sky together would be 1.6 ; that is, it would require $3 \frac{3}{4}$ seconds exposure to sun and sky together to produce the unit tint of measurement in the same paper, which calculated value could be immediately verified by another strip cut from the same paper. I have repeatedly made such verifications, which give me a test of the degree of accuracy with which tints can be matched.

By comparing a strip thus gradually shaded in proportion to the time, with another strip shaded as the inverse square of the distance from a near source of light, it is readily shown that the effect of a light acting at the distance unity for four seconds is equal to that of the same light acting at the distance $\frac{1}{2}$ for one second.

Observations of these ratios can be made with any uniform piece of paper from which the strips are cut. In the early experiments at Dacca I prepared sheets of sensitized paper with great care. I have found, in later experiments at Dacca and in Somersetshire, that any sheet of fairly good photographic paper gives satisfactory results; i.e. the error introduced by

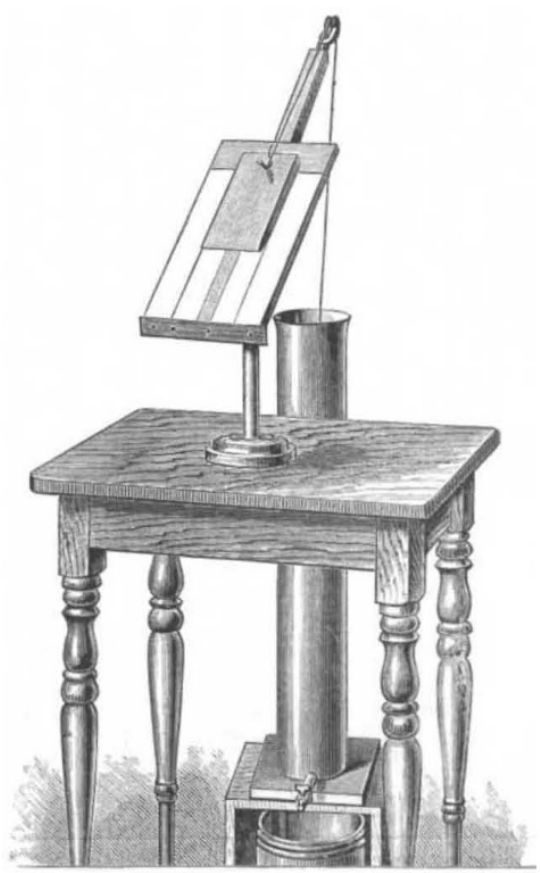

Water-motion actinometer.

want of uniformity in one sheet of paper is inappreciable in comparison with the margin of possible error in the "reading," i.e. the matching of tints.

I originally took as my unit of absolute measurement a particular tint produced in a piece of particular paper by a Dacca candle. I found that at an altitude $\alpha$ of the sun this tint was produced (by the effect of the sun alone) in the same paper in (say) 16 seconds, i.e. the effect of the sun alone at altitude $\alpha$ was represented by the number $\frac{1}{16}=0.0625$. By the system of ratios, I found the corresponding numbers for all altitudes $10^{\circ}$ to $45^{\circ}$. These ratios were the means of very numerous observations ex. tending over several cold seasons at Dacca where I had a perfect sky for months. If at any time I wish to recover the Dacca unit of measurement in any particular paper, I take a sun strip (at any altitude of the sun, $\gamma$ ) in the water-motion actinometer; I see in my table (B) that for the altitude $\gamma$ the effect of the sun alone is (say) 0.12; I make a mark on my sun strip at the point where it has been exposed $8 \frac{1}{3}$ seconds; the effect at that point in that paper is my Dacca unit.

My English observations have been taken in this manner without any reference to a candle. The observations of Sir H 